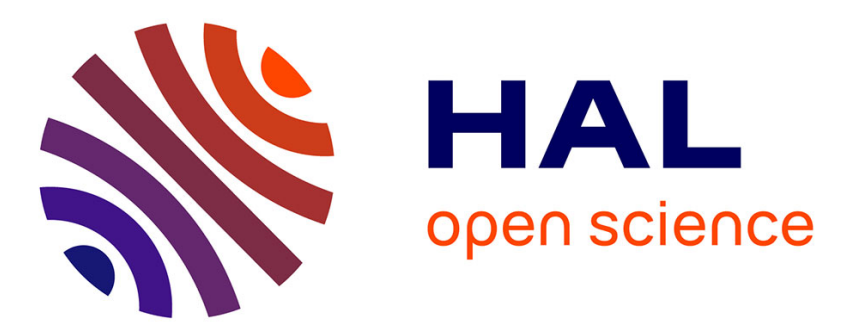

\title{
Problématique archéologique des carrières antiques en Gaule
}

\author{
Jean-Claude Bessac, Robert Sablayrolles
}

\section{To cite this version:}

Jean-Claude Bessac, Robert Sablayrolles. Problématique archéologique des carrières antiques en Gaule. Gallia - Archéologie de la France antique, 2002, Carrières antiques de la Gaule, 59, pp.39. 10.3406/galia.2002.3092 . hal-01911761

\section{HAL Id: hal-01911761 \\ https://hal.science/hal-01911761}

Submitted on 20 Jan 2020

HAL is a multi-disciplinary open access archive for the deposit and dissemination of scientific research documents, whether they are published or not. The documents may come from teaching and research institutions in France or abroad, or from public or private research centers.
L'archive ouverte pluridisciplinaire HAL, est destinée au dépôt et à la diffusion de documents scientifiques de niveau recherche, publiés ou non, émanant des établissements d'enseignement et de recherche français ou étrangers, des laboratoires publics ou privés.

\section{(ㅇ)(1) $\$$}

Distributed under a Creative Commons Attribution - NonCommercial - NoDerivatives| 4.0 


\title{
PROBLÉMATIQUE ARCHÉOLOGIQUE DES CARRIÈRES ANTIQUES EN GAULE
}

\author{
Jean-Claude BessaC et Robert SABLAYROLles
}

La construction en dur a toujours été considérée comme un des apports majeurs de la présence romaine dans les provinces occidentales de l'Empire, si l'on ex:epte quelques points de la bordure méditerranéenne. $S_{\text {E }}$ dans bien des domaines, comme, par exemple, l'exploitation du terroir agricole ou l'extraction du minerai et la fabrication du métal, les recherches récentes notent en évidence la richesse des techniques et 1'minpleur des travaux des périodes préromaines, il ne fait b. de doute que l'extraction de la pierre, bien qu'elle n'ait pas été, naturellement, inconnue des populations protohistoriques, constitua, lors de l'expansion romaine, une mutation économique essentielle. Le développement des villes et de leurs centres civiques et religieux monumentaux, le passage progressif de l'habitat de terre et de bois à la domus en pierre, l'expansion, dans le monde rural, du phénomène de la villa, qui contamina les fermes en construction légère, la multiplication des agglomérations secondaires autour des pôles économiques, les grands chantiers que constituaient aqueducs, remparts ou édifices de spectacles créèrent, dans le monde urbain comme dans le monde rural, une formidable dynamique de construction, qu'il fallut approvisionner à la hauteur de ses gigantesques besoins. À un degré moindre, mais d'une manière tout aussi nouvelle et tout aussi spectaculaire, l'acculturation, qui rendit commun le recours au support épigraphique, dans le domaine édilitaire comme dans le domaine funéraire, et l'enrichissement des aristocraties, qui multiplia les éléments de décor architectural ou statuaire, contribuèrent à développer l'économie de la pierre.

\section{RECHERCHES ANTÉRIEURES ET PROBLÉMATIQUE}

\section{DES GRANDES SYNTHÈSES AUX ÉTUDES PONCTUELLES}

L'importance du phénomène n'a sans doute jamais échappé aux chercheurs, comme en témoignent les travaux pionniers de C. Dubois (1908), qui proposa une première synthèse sur le sujet pour l'ensemble du monde romain. C'est souvent à cette très large échelle que le thème fut traité ensuite par ses successeurs. Parmi eux, J. B. Ward-Perkins, entre 1933 et 1981, consacra son œuvre plus particulièrement aux marbres; ses écrits ont fait l'objet d'un recueil récent (Dodge, Ward-Perkins, 1992). Une autre synthèse, peu diffusée malgré la remarquable pertinence de ses analyses, mérite d'être mentionnée: Quarries in Roman Provinces d'A. Dworakowska (1983), qui complète son premier ouvrage, Quarries in Ancient Greece (1975), tout aussi intéressant. Mais, pour la Gaule proprement dite, il fallut attendre 1984 pour disposer de l'essentiel de la documentation disponible sur les carrières dans un ouvragc dc R. Bedon (1981). Malgré l'utilité et l'intérêt de toutes ces synthèses, aucune ne fut élaborée à partir des résultats de véritables fouilles archéologiques effectuées dans les carrières. Le premier chercheur à avoir pris cette voie fut J. Röder qui, entre 1951 et 1969, publia le fruit de ses investigations de terrain, conduites principalement dans les carrières de Rhénanie. Ses recherches sur les carrières le conduisirent également en Tunisie, en Égypte et en Turquie, 


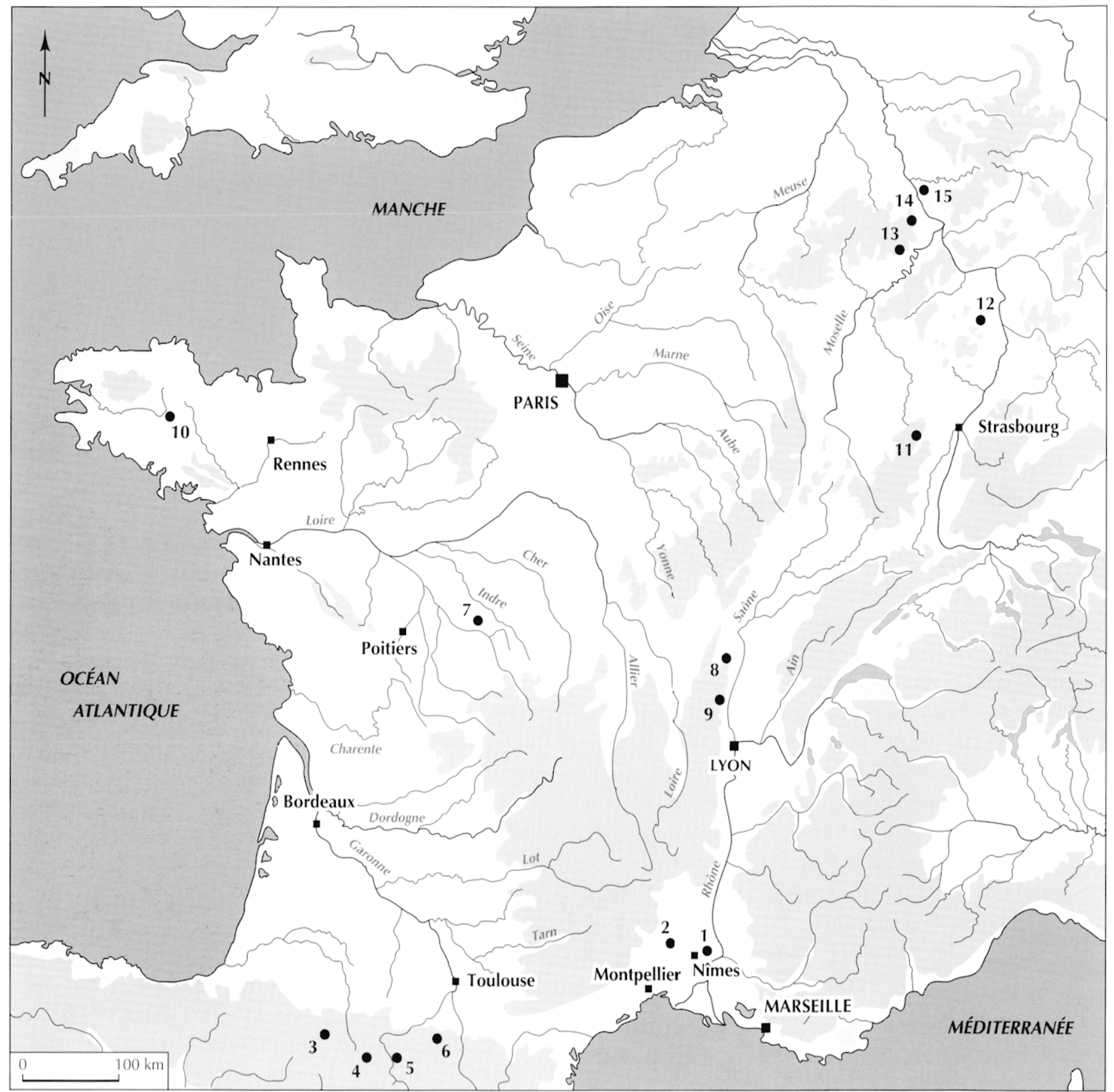

Fig. 1 - Localisation des carrières décrites dans ce dossier: 1, LWstel (Gard) ; 2, Le Bois des Lens (Gard); 3, carrières du massif du Béonul, Lourdes (Hautes-Pyrénées) ; 4, carrières de marbre des vallées de l'Aure et de l'Adour; 5 , carrières de marbre de la vallée de la (jurvnne; 6, carrières de marbre de l'Ariège; 7, Tendu (Indre) ; 8, Saint-Boil (Sâone-et-Loire) ; 9, La Lie à La Roche-Vineuse (Saône-et-Loire) ; 10, carrière de granite de Locuon en Ploërdut (Morbihan); 11, carrières de grès de La Croix-Guillaume à Saint-Quirin (Moselle); 12, carrière de grès de Kriemhildenstuhl; 13, carrière souterraine de tuf volcanique près de Kruft; 14, carrière de tuf volcanique de Brohl; 15, carrière de trachyte de Drachenfels (fonds de carle (C) M. Feugère et M. Py, CNRS, 1993).

mais ne sont présentés en détail ici, dans la contribution de D. Lukas (p. 155-174), que les travaux relatifs au nordest de la Gaule. Ces vingt dernières années, plusieurs autres auteurs ont proposé quelques résultats partiels sur la question des carrières de Gaule, mais aucunc nouvelle synthèse nationale n'est parue (Braemer éd., 1986; 
Lorenz, Benoît éds, 1991 ; Duval, Christe dir., 1993 ; Lorenz dir., 1993, 1996 ; Cabanot et al. éds, 1995 ; Bessac, 1996 ; Lorenz et al. dir., 2000). Le caractère ingrat de la recherche et la spécificité de l'approche technique, qui sont communs au monde des carrières comme à celui de toutes les exploitations de ressources naturelles (bois, minerai, etc.), ont cependant souvent découragé les chercheurs ou les ont cantonnés dans l'analyse des matériaux de luxe et, parfois, les ont entraînés, devant les maigres résultats de leurs efforts, à prêter trop généreusement à l'Antiquité des traces d'extraction, qu'aucun argument sérieux ne permettait de dater.

\section{PROBLÈMES D'IDENTIFICATION ET DE DATATION}

Il est vrai qu'une des difficultés majeures de l'étude des carrières, difficulté qu'elle partage avec l'archéologie des mines, est celle de l'identification et de la datation. La reprise, à diverses époques, de l'exploitation d'un matériau fait disparaître, par la progression des fronts de taille ou par l'accumulation des déblais, les vestiges antiques, réduits à l'état d'images virtuelles ou enfouis sous des tonnes de déchets d'extraction et de taille plus récents. Cette situation peut décourager l'entreprise archéologique, car, dans bien des cas, elle exige de considérables travaux d'approche pour un résultat aléatoire : la carrière est, en effet, souvent, un lieu austère, où il est très difficile de localiser les quelques mètres carrés d'abri sous les milliers de mètres cubes de déchets d'extraction. En montagne, il est rare que le carrier y séjourne et il n'a donc guère l'occasion d'abandonner des objets de la vie quotidienne, qui deviennent, des siècles plus tard, les fossiles directeurs de l'archéologue. Remuer des milliers de tonnes de déblais pour découvrir un sol de carrière qu'il sera impossible de dater est donc une perspective qui incite à la prudence, même si le présent dossier montre qu'il existe, dans ce domaine, de nombreuses exceptions d'un apport remarquable.

Cette difficulté à identifier et à dater, fréquente malgré tout, devrait entraîner une rigueur dans la méthode, qui, nous l'avons dit, n'a pas toujours été de mise. L'enthousiasme du chercheur ne l'autorise pas à restituer, en avant des fronts de taille* récents, des fronts de taille

* Les mots suivis d'un astérisque se trouvent dans le glossaire, p. 189-194. antiques virtuels, et il faut réserver le qualificatif de galloromain aux carrières qui ont effectivement fourni du mobilier archéologique, ou dont le matériau est suffisamment caractéristique pour pouvoir être, sans risque d'erreur, identifié sur des sites antiques. Si ce principe appauvrit quantitativement la cartographie souvent trop généreuse des vestiges antiques d'extraction, les possibilités offertes par l'exploration archéologique n'en demeurent pas moins d'une exceptionnelle richesse, comme l'illustre le présent dossier, qui réunit les recherches récentes en la matière dans les provinces gauloises et germaines de l'Empire romain (fig. 1). Ces recherches de terrain, en particulier pour le marbre, sont souvent le fruit de la reprise des exploitations par l'industrie moderne, constatation qui doit amener à une vigilance rigoureuse des services compétents comme des amateurs: ce sont les déblaiements des entreprises contemporaines d'extraction qui seront, dans ces domaines, les pourvoyeurs des recherches archéologiques dans les carrières antiques. Le tableau, là non plus, ne doit pas être noirci à l'excès : les exceptions, qui ont engendré fouilles programmées ou sauvetages urgents, demeurent nombreuses, surtout pour les matériaux de construction communs, dont les carrières ont été plus souvent épargnées par les réoccupations récentes. Leur richesse potentielle tient dans la diversité des domaines abordés, comme en témoignent les divers exemples réunis ici : aspect technique de l'extraction, du geste du carrier à la stratégie du maître d'œuvre de la carrière; vie quotidienne des exploitants, dans leur rythme de travail comme dans leurs préoccupations tant matérielles que spirituelles; commercialisation du produit, brut ou préparé, par voie d'eau ou voie de terre, à destination proche ou lointaine; influence de l'économie de la pierre dans l'environnement paysager, économique, social, culturel.

\section{DES APPROCHES TECHNIQUES PRÉPONDÉRANTES}

\section{LES ROCHES}

Les aspects techniques de la recherche archéologique en carrière sont essentiels et touchent plusieurs secteurs. Le premier concerne la roche, son esthétique (couleur, veinage, possibilités de polissage), ses caractéristiques techniques dans la carrière comme dans le processus de 
transformation (élaboration de matériaux de construction, d'objets rituels ou funéraires, sculpture, épigraphie, etc.). Si la définition géologique de la pierre est indispensable aux recherches sur la diffusion de la production de la carrière, la caractérisation du matériau du point de vue des spécialistes de la construction (bâtiments, travaux publics) est tout aussi importante pour pouvoir apprécier les choix des carriers antiques en matière d'implantation et de stratégie de progression de leurs exploitations. Les Romains allaient, par exemple, extraire à plus d'une centaine de kilomètres de son lieu d'emploi un certain sous-faciès oolithique de calcaire fin, en raison des avantages sculpturaux spécifiques à cette structure, alors que, dans la même formation géologique, ils négligeaient le faciès dominant, tout aussi fin mais plus cassant. Le jeu des fissures de la roche, son pendage, ses veines, ses variations de teinte, sa fissilité, son taux de déchets et, d'une manière générale, son comportement sous l'outil du carrier, du tailleur de pierre et du sculpteur, orientaient les choix de l'exploitant antique. Même s'il existe des règles générales qui s'appliquent à chacune des grandes familles de roches (calcaire, grès, granite, marbre, etc.), chaque site d'extraction constitue un cas particulier dans ce domaine.

\section{STRATÉGIES D'EXPLOITATION DES CARRIÈRES}

La stratégie d'implantation et la progression de la carrière antique dépendaient aussi de facteurs extérieurs à la roche : climat, topographie du lieu, réseau local des voies (terrestres, fluviales et maritimes), importance du chantier de construction à l'origine de la commande, statut économique, social et juridique des carriers, équipement en matière de levage et de transport. Il est sûr qu'une petite équipe de carriers romains, œuvrant sur la bordure méditerranéenne dans du calcaire coquillier pour construire un modeste sanctuaire rural, adoptera des choix stratégiques totalement différents de ceux d'une forte escouade de légionnaires-carriers de la Gaule de l'Est, chargée d'approvisionner en pierre une grande agglomération. Cela reste vrai même si la roche exploitée offre des caractéristiques similaires, et les différences seront encore plus marquées si la comparaison concerne des phases distinctes de l'Antiquité.

Les questions de stockage, bardage, levage et débardage des pierres d'une carrière romaine sont diffi- ciles à aborder en l'absence de fouilles. Trop souvent, les hypothèses qui sont proposées à ce sujet ne font que reprendre les reconstitutions graphiques des diverses éditions historiques de Vitruve, sans tenir compte du fait que cet architecte et théoricien romain, comme ses traducteurs et commentateurs modernes, traitait de la construction monumentale, et non pas des carrières. Même s'il existe des points communs entre les deux activités, des facteurs importants de différenciation, en particulier la conformation générale de l'exploitation et sa disposition par rapport au relief et à l'eau, obligent à n'user qu'avec prudence du raisonnement par analogie. Les déblaiements de carrières antiques, surtout lorsqu'ils sont effectués dans le cadre de fouilles archéologiques, mettent parfois au jour des traces d'engins d'exploitation : trous d'implantation d'appareils de levage, traces d'usure dues au cordage d'un cabestan, ornières de chariots. Parfois, le témoignage est encore plus concret comme les aires de stockage, les quais de chargement, les rampes d'accès et les canaux de desserte. Mais ces traces et ces vestiges sont souvent impossibles à dater sans l'étude du comblement qui les scelle.

\section{LES TRACES D'OUTILS : UNE CLEF TECHNIQUE, CHRONOLOGIQUE ET ANTHROPOLOGIQUE}

Le dernier aspect technique, certainement un des plus importants dans les investigations archéologiques en carrières, concerne les traces d'outils. Un triple objectif caractérise ce domaine de la recherche : l'identification des outils, la chronologie de leur utilisation, leur interprétation dans un esprit d'analyse anthropologique, sociale et culturelle du chantier d'extraction.

L'identification des outils, grâce à leurs impacts sur la roche, implique un minimum d'apprentissage pour les archéologues. Il faut être conscient que, selon la finesse de la pierre et son degré d'altération superficielle, les risques de confusion entre traces d'outils et déformations naturelles augmentent sensiblement. Un calcaire coquillier alvéolaire sera toujours plus difficile à lire qu'un calcaire ou un grès à grains fins, et il est parfois impossible de savoir quels outils ont été utilisés. Dans l'analyse de l'outil proprement dit, il est toujours plus facile d'identifier la forme de son extrémité active que de reconnaître ses lignes générales, son volume et son poids. En l'absence de découverte de l'outil lui-même, en contact ou en corré- 
lation directe avec ses traces, sa description ne peut être qu'hypothétique. Souvent, cependant, les enquêtes ethnoarchéologiques et l'archéologie expérimentale permettent de réduire la marge d'erreur.

La datation des traces pose d'autres problèmes. Identifier des impacts d'outils sur des fronts ou des sols de carrière sans avoir de certitude sur leur chronologie ne présente qu'un intérêt limité. La question se résout parfois par la découverte d'inscriptions en relation sûre avec les traces observées, mais il s'agit là d'une situation fort rare en dehors des carrières exploitées par l'armée. La fouille stratigraphique de zones de comblement peut également fournir des témoins chronologiques, sous la forme de fossiles directeurs, notamment céramiques. La difficulté de cette entreprise réside dans le choix judicieux des emplacements de fouille au sein des comblements des anciennes exploitations (Bessac, 1986b, p. 160-165 et 1999a, p. 35-36). Le nombre des sites où une exploration de ce type peut être appliquée est généralement assez restreint, mais, une fois que les jalons d'une chronologie typologique des techniques sont ainsi mis en place, il est souvent possible de dater des sols et des fronts libres de comblement. Il n'existe pas, à l'heure actuelle, d'autre procédé, archéométrique notamment, qui permette de dater sûrement des structures rocheuses taillées.

L'interprétation anthropologique des impacts d'outils sur la roche requiert une bonne connaissance des pratiques traditionnelles du métier de carrier et des professions de la pierre en général. Il s'agit d'évaluer le niveau professionnel des carriers, leur productivité, leur nombre et leur mode de fonctionnement en équipe. Les indices utilisés pour cela sont la densité des impacts d'outils, leur sens, leur dispersion et leur ampleur. Par exemple, une série d'impacts de pics dispersés sur le socle rocheux a une autre signification que les mêmes traces alignées sur un front : dans le premier cas, il s'agit certainement d'une rectification des irrégularités de la roche, tandis que, dans le second, c'est vraisemblablement la matérialisation d'un repérage d'une microfissure peu visible. C'est la même catégorie d'observations qui permet de distinguer les gauchers des droitiers, de savoir combien de carriers travaillaient simultanément à l'extraction d'un bloc ou dans un secteur déterminé de la carrière. Elle permet enfin de reconnaître, dans la diversité des traces, ce qui relève de coups de main personnels de ce qui résulte, au contraire, de variations typologiques liées à une évolution technologique.

\section{LES CARRIERS}

La carrière n'est pas simplement un lieu de travail, où se posent des questions d'extraction et de bardage de blocs, d'évacuation de déchets et de rentabilité dans la progression. Elle est aussi un lieu de vie, où se mêlent préoccupations matérielles du quotidien comme interrogations culturelles, voire spirituelles. Un simple examen attentif des vestiges techniques peut déjà offrir une ouverture sur les rythmes de travail, saisonniers ou continus, liés à une commande ponctuelle ou à une demande plus permanente. Dans ces différents cas de figure, la vie comme la nature des professionnels de la pierre ne sont pas identiques. Des observations analogues peuvent se déduire de la mise en évidence d'activités diversifiées, comme la présence d'ateliers d'ébauche ou de façonnage plus élaboré des pièces. Cette diversification du travail induit une différenciation de qualification des ouvriers, qui a de bonnes chances de se traduire par une différenciation sociale et économique. Il est difficile d'aller plus loin en la matière si on ne dispose pas d'arguments épigraphiques pour analyser la condition juridique des individus. La carrière est parfois, dans ce domaine, un lieu privilégié. Avec l'exploitation de la pierre et sa diffusion vers les milieux urbains ou aristocratiques pouvait se développer, dans la carrière elle-même, une acculturation à la pratique épigraphique. Les inscriptions comme les représentations iconographiques n'y sont pas rares, révélant le rôle de l'armée en Rhénanie, la participation active d'une population indigène dans les exploitations marbrières des Pyrénées, l'alphabétisation souvent maladroite de carriers. On note cependant d'importantes différences selon la nature de la roche exploitée, les régions et, vraisemblablement, les périodes. Ainsi, malgré le nombre relativement élevé d'opérations archéologiques dans les carrières antiques méditerranéennes, aucune inscription ni sculpture n'y a été découverte.

\section{LA DIFFUSION DES PIERRES}

\section{LES VOIES DU TRANSPORT}

La diffusion du produit constitue un volet essentiel de l'économie de la pierre. Elle peut être locale, limitée dans l'espace comme dans le temps à une commande 
telle qu'édification de rempart ou aménagement d'aqueduc, ou plus lointaine, dans le cas de produits de luxe acheminés vers les marchés régionaux ou, dans certains cas, jusqu'aux extrémités de l'Empire. La première question, dans ce domaine, est celle du transport, que peut éclairer la découverte de vestiges tels que pont ou voie. Si le bardage appartient encore aux contraintes techniques de la carrière proprement dite, la diffusion empruntait ensuite les voies fluviales, souvent privilégiées, mais aussi les voies terrestres, qui n'étaient pas négligées. La hiérarchisation de ces deux moyens de transport n'admet pas de réponse univoque : elle était fonction de la distance, du poids, mais aussi de contraintes topographiques et, sans aucun doute, de considérations économiques, voire politiques. Le marbre pouvait ainsi, vraisemblablement, franchir les Pyrénées sous forme de placage, alors que les grosses pièces ou les pierres de construction empruntaient plutôt les voies fluviales, grâce, le plus souvent, à la radellerie ', étant donné le débit modeste de nombreux cours d'eau.

\section{LE RÔLE DE L'ARCHÉOMÉTRIE}

L'étude de la diffusion, qu'elle soit locale, régionale ou lointaine, suppose une identification assurée du matériau et de sa carrière d'origine. Dans ce domaine, qui requiert la contribution fondamentale des géologues et des pétrologues, rigueur et prudence sont de mise. Le temps est révolu de l'archéologue omniscient, capable au premier coup d'œil de déterminer l'origine d'un marbre blanc ou de rattacher à telle carrière, distante de plusieurs dizaines de kilomètres, les moellons d'un rempart. Si la collaboration avec les géologues est désormais une nécessité admise par tous, on ne saurait, cependant, verser dans un positivisme excessif et imaginer une science salvatrice, dont les méthodes sophistiquées seraient une garantie de fiabilité et d'absolu. Les expériences récentes montrent les apports mais aussi les limites des techniques scientifiques d'identification : la recherche, dans ce domaine comme dans les autres, est une lente progression qui suppose collaboration et échanges, modestie et remises en question. L'archéométrie, qui a fait ses preuves, demeure une technique, pas un dogme.

1. Activité de transport par radeau.

\section{L'IMPORTANCE DE CORPUS CLAIREMENT DÉFINIS}

Malgré les marges d'incertitude et d'erreur que comportera donc toujours l'identification des matériaux, et notamment des matériaux de luxe, l'analyse de la diffusion, si elle s'en tient aux acquis assurés, constitue un pan indispensable de l'histoire des carrières en les incluant dans les problématiques de l'histoire économique de l'Antiquité. Pour être utilisable, une base de données en la matière, au-delà de sa fiabilité, doit présenter sens et valeur statistique. Il serait vain de raisonner sur tel ou tel individu isolé, même si ce type de démarche peut inférer la diffusion à longue distance de certains produits. Il faut constituer des tableaux statistiques sur des groupes signifiants: ensemble des pièces d'une agglomération, d'une villa, d'un édifice, dont les limites et les phases chronologiques sont bien déterminées. Ce groupe doit ensuite être inséré dans une série qui, seule, lui donnera sa véritable signification. Ce n'est qu'en possession de ces outils que l'on pourra raisonner sur des circuits d'approvisionnement, sur des lois de marché, sur des phénomènes de mode, par essence variables dans le temps comme dans l'espace, tous éléments qui ont joué un rôle dans l'apparition, le développement et la récession des carrières antiques.

\section{L'INSERTION DES CARRIÈRES DANS LEUR ENVIRONNEMENT}

Insérées dans les problématiques et les analyses des grands courants économiques du monde antique, les carrières doivent l'être également dans leur environnement archéologique et historique immédiat. Nous avons déjà souligné l'évidence des mutations que constituèrent, pour les provinces occidentales, le développement de la construction en dur et les nécessités d'approvisionner les grands chantiers. Ces mutations, si elles affectèrent les destinataires comme les producteurs de la pierre, se ressentirent également dans l'environnement proche des carrières. Elles furent d'abord économiques et sociales, puisqu'elles inclurent dans des circuits d'échanges parfois à grande échelle des régions souvent isolées et mal accessibles. Sur le plan de la démographie, du regroupement des communautés, des ressources dont elles disposaient, il va de soi que les collectivités proches 
des carrières changèrent dans leur taille comme dans leurs habitudes. Au-delà de ces changements dans les structures comme dans la vie quotidienne des populations, l'activité extractive, les ateliers de transformation, les échanges avec les chantiers de construction parfois lointains créèrent, dans l'environnement de lieux d'extraction souvent reculés, ce qu'on pourrait appeler une culture de la pierre. Cette culture, qui mettait à la disposition des communautés proches des carrières le matériau sous toutes ses formes, les initiait en même temps aux cadres idéologiques de son utilisation: pratiques épigraphiques, iconographiques, sociales, funéraires ou religieuses. Vecteur de changements économiques et sociaux, le développement des carrières était ainsi, en même temps, un facteur d'acculturation. Percevoir ces réalités suppose de replacer les carrières dans une carte archéologique régionale.

La matière, on le voit, est riche et polymorphe : elle ne saurait être épuisée dans une synthèse comme celle qui est présentée ici, qui se veut simplement un bilan des acquis récents, mesuré à l'aune des problématiques diverses qui peuvent être appliquées à l'étude des carrières des provinces gauloises et germaines de l'Empire romain. 